\title{
Model Discrimination and Parameter Estimation via Infeasibility Certificates for Dynamical Biochemical Reaction Networks
}

\author{
Steffen Borchers ${ }^{*, * *}$ Philipp Rumschinski* Sandro Bosio ${ }^{* * *}$ \\ Robert Weismantel ${ }^{* * *}$ Rolf Findeisen* \\ * Institute for Automation Engineering, Otto-von-Guericke Universität \\ Magdeburg, Germany \\ ** Max Planck Institute for Dynamics of Complex Technical Systems, \\ Magdeburg, Germany \\ *** Institute for Mathematical Optimization, Otto-von-Guericke \\ Universität, Germany
}

\begin{abstract}
Current approaches to parameter estimation and model invalidation are often inappropriate for biochemical reaction networks. This is because often only noisy measurements and sparse experimental data is available, and since they do not take the special structure of biochemical reaction networks into account.

In this work a new method to prove model invalidity and to estimate parameters is introduced. It is based on a certificate of non-existence of feasible parameterizations for a given models. This is done by reformulating the model invalidation task into a set-based feasibility problem. As shown, due to the polynomial structure of many biochemical reaction systems, it is possible to relax the non-convex feasibility problem into a semidefinite program and thus to obtain conclusive results on model invalidity and parameter estimation. Our framework allows us to consider the arising difficulties posed by biochemical reaction networks by taking the specific structure of the dynamics and model outputs into account. It also enables us to discard large parameter regions as infeasible. We also show on a well-known biological example, namely the Michaelis-Menten and the Henri kinetics, how with this method it is possible to discriminate between model hypotheses and how to estimate parameters.
\end{abstract}

\section{INTRODUCTION}

In systems biology, modeling is an important tool for analysis and prediction of metabolic and signal transduction processes. Limited prior knowledge of the involved reaction mechanisms and system structure, however, often results in competing hypotheses, where the kinetic parameters are often completely unknown or may vary within one order of magnitude. For a successful analysis, it is important to discriminate between the alternatives, taking the parameter uncertainty into account, so as to obtain a model that captures the essential behavior of the process under study.

Model validation consists of checking whether a model is able to represent a given observed experimental behavior. However, as is well known (e.g. Epstein and Pojman [1998], Prajna [2006]), model validation is a misleading term. Indeed, it is impossible to prove that a model or reaction mechanism is correct, as to do so an infinite number of experiments would be required. Thus, the purpose of model validation techniques is rather to invalidate a model by proving inconsistency of the model hypotheses with the experimental data.

Traditional approaches to model validation include exhaustive Monte Carlo simulations to check for consistency with experimental data (Robert and Casella [2004]). Indeed, simulation is a way to test whether a model can reproduce some characteristics of the process under study.
However, the computational demands for proving inconsistency are in general prohibitive, and such a proof by simulation is typically not exact.

One of the first works of model validation in the context of invalidation was given by Smith and Doyle [1992] using frequency-domain data. Chen and Wang [1996] and Poolla et al. [1994] extended this result so as to consider also time-domain data. The applicability of these approaches is however limited to linear models.

Evans et al. [2004] and Schnell et al. [2006] proposed an analytical approach to extend model distinguishability to nonlinear models. It involves the construction, when possible, of a smooth mapping between the two candidate models (Evans et al. [2004]). The applicability of this method is however restricted in practice to very small systems.

Quite recently, Prajna [2006] proposed a model invalidation framework using barrier certificates, which are functions of state-parameter-time. These time barriers separate possible model trajectories and measurement data, allowing to conclusively invalidate a model. Finding such barrier certificates however is a nontrivial task. Moreover, the existence of a suitable barrier function cannot be guaranteed for all invalid models. 
Our work is based on the work of Kuepfer et al. [2007], which introduced a semidefinite programming approach for parameter estimation based on stationary measurements. By using a different class of infeasibility certificates for polynomial reaction mechanisms similar to the one proposed in Waldherr et al. [2008] for sensitivity analysis, the method we proposed allows to consider a large class of nonlinear systems subjected to bounded, but possibly large, parameter uncertainties and measurement errors. Different candidate models can be discriminated by proving inconsistency with the available data while taking parameter and measurement uncertainties into account.

The proposed approach also lays the basis for model parameter estimation. By invalidating subsets of the parameter space it is indeed possible to classify the admissible parameter region into feasible regions that explain the measurements and infeasible regions that do not.

This paper is structured as follows: in Section 2 the invalidation setting is considered, the basic results using feasible parameter sets are presented, and the key ideas of the proposed model discrimination and parameter estimation technique are outlined. Then, in Section 3 we show how to solve the resulting feasibility problem via a convex semidefinite program, and how the feasible parameter sets can thus be estimated. In Section 4 we provide examples on model discrimination and parameter estimation. Our approach is summarized and discussed in Section 5 .

\section{MODEL INVALIDATION AND PARAMETER ESTIMATION}

The basis for the modeling of biochemical reaction networks is the description of the occurring reactions in the form

$$
\alpha_{1} S_{1}+\cdots+\alpha_{n_{s}} S_{n_{s}} \rightarrow \beta_{1} O_{1}+\cdots+\beta_{n_{p}} O_{n_{o}} .
$$

Hereby the substrates $S_{i}$ are processed to the output products $O_{i}$, while the pre-factors $\alpha_{i}, \beta_{i}$ define the stoichiometric relations of the participating compounds.

Such biochemical reaction networks can be expressed in terms of ordinary differential equations if spacial and stochastic effects are neglected. The dynamic models can then be defined considering the reaction fluxes and the corresponding balance equations as

$$
\dot{x}=N \nu(x, p),
$$

where $x \in \mathbb{R}^{n_{x}}$ denotes the vector of concentrations, $p \in \mathbb{R}^{n_{p}}$ the vector of kinetic parameters, and $\nu$ the vector of fluxes. The stoichiometric matrix $N$ is built up from the factors $\alpha_{i}, \beta_{i}$.

There are many possibilities to describe the reaction fluxes (see e.g. Cornish-Bowden [2004]), including the law of mass action, Hill kinetic, and Monod kinetic, the first approach being one of the most frequently used. Hereby, the reaction fluxes are proportional to the substrates, resulting in system (1) with polynomial right-hand side. As our approach is based on this special structure, we focus on mass action models.

Describing this model with a suitable discretization method we obtain the following discrete time model

$$
\Sigma=\left\{\begin{array}{l}
x[k+1]=g(x[k], p), \\
y[k]=h(x[k], p)
\end{array}\right.
$$

where $k \in \mathbb{N}$ is the time index, $x[k] \in \mathcal{X} \subset \mathbb{R}^{n_{x}}$ the vector of state variables, $y[k] \in \mathbb{R}^{n_{y}}$ the model output, and $p \in \mathcal{P} \subset \mathbb{R}^{n_{p}}$ the parameter vector. In the remainder we consider $g(\cdot, \cdot)$ and $h(\cdot, \cdot)$ to be polynomial functions. Note that this is a mild assumption, since a large class of systems biology models is based on mass action kinetics.

Remark 1. This setting can be easily extended to rational functions, as well as to consider model inputs.

Furthermore, let an experiment be performed with the real process, and let a sequence of (noisy) measurements $\mathcal{Y}=\left\{\mathcal{Y}_{k_{0}}, \mathcal{Y}_{k_{1}}, \ldots, \mathcal{Y}_{k_{N}}\right\}$ be taken at times $k_{0}, \ldots, k_{N}$, with $\mathcal{Y}_{k_{i}} \subset \mathbb{R}^{n_{y}}$ for every $i \in\{0, \ldots, N\}$. We also assume that the state variables are always in the feasible set $\mathcal{X}$, i.e., $x[k] \in \mathcal{X}$ for all $k \in\left\{0, \ldots, k_{0}, \ldots, k_{N}\right\}$.

Considering this setup we aimed to solve the model invalidation and parameter estimation task. The model invalidation problem can then be formulated as follows:

Problem 2. (Model Invalidation). Given a model (2), an admissible parameter set $\mathcal{P}$, and a measurement sequence $\mathcal{Y}$, find a feasible parametrization for $\Sigma$, i.e., a parameter $p \in \mathcal{P}$ for which the model (2) can reproduce an output trajectory $y[k]$ such that $y\left[k_{i}\right] \in \mathcal{Y}_{k_{i}}$ for every $i \in$ $\{0, \ldots, N\}$ for some initial condition, or prove that none exists.

Note that, in case of a measurement process involving a stochastic error, it is possible for the actual output to be missed by the respective measurements. In this case it can happen to regard a valid model as invalid, or to give an incomplete parameter estimation.

Traditional approaches to solve Problem 2 include exhaustive Monte Carlo based simulation using random parameters and initial conditions sampled respectively from $\mathcal{P}$ and $\mathcal{X}_{0}$. If no trajectory touching the measurements is found after many simulations, then inconsistency is (statistically) concluded. Increasing the number of simulations may improve the reliability of this approach, but it remains inherently not exact.

Our approach to prove model invalidity is based on a certificate of non-existence of feasible parametrizations. Remarkably, this is achieved without direct simulating nor computing the solution of the model. Moreover, uniqueness of the solution of the discrete system is not required, although such a property often holds for most physical models.

\subsection{Proposed Framework}

The key idea is to reformulate the invalidation problem as a feasibility problem. While taking into account the measurements and parameter bounds as constraints, infeasibility of the feasibility problem can be certified for the considered system class as shown later on. Thus, model invalidity can be proved.

Two Measurements Let us start considering the simple case of two measurements $\mathcal{Y}_{k}, \mathcal{Y}_{k+1}$ taken at consecutive time indexes; we define their feasible parameter set as the set $\mathcal{P}_{k, k+1}=\{p \in \mathcal{P}: \exists x \in \mathcal{X} \mid h(x, p) \in$ $\left.\mathcal{Y}_{k}, h(g(x, p), p) \in \mathcal{Y}_{k+1}\right\}$. We can then show that following theorem holds: 
Theorem 3. (Model Invalidity): Given a model (2), a parameter set $\mathcal{P}$, and two consecutive measurements $\mathcal{Y}_{k}, \mathcal{Y}_{k+1}$, the model $\Sigma$ can be valid for the real system under study only if $\mathcal{P}_{k, k+1} \neq \emptyset$.

As a consequence, if $\mathcal{P}_{k, k+1}=\emptyset$ then we can say that the model (2) and its admissible parameter set $\mathcal{P}$ are invalidated by the measurements $\left\{\mathcal{Y}_{k}, \mathcal{Y}_{k+1}\right\}$.

The proof of Theorem 3 is simply done by contradiction. Suppose that a feasible parametrization $p^{*} \in \mathcal{P}$ for $\mathcal{Y}=\left\{\mathcal{Y}_{k}, \mathcal{Y}_{k+1}\right\}$ exists, while $\mathcal{P}_{k, k+1}=\emptyset$. By definition of feasible parametrization, there exists a trajectory for which $y[k]=h(x[k], p) \in \mathcal{Y}_{k}$ and $y[k+1]=h(x[k+1], p) \in$ $\mathcal{Y}_{k+1}$, with $x[k+1]=g(x[k], p)$. It is then straightforward to see that $p^{*} \in \mathcal{P}_{k, k+1}$, contradicting $\mathcal{P}_{k, k+1}=\emptyset$.

As measurements are not necessarily taken at consecutive time steps, let us extend the above definition to a pair of arbitrary measurements $\mathcal{Y}_{k}$ and $\mathcal{Y}_{k+m}$. We can then extend define the feasible parameter set as $\mathcal{P}_{k, k+m}=\{p \in$ $\left.\mathcal{P}: \exists x \in \mathcal{X} \mid h(x, p) \in \mathcal{Y}_{k}, h\left(g^{m}(x, p), p\right) \in \mathcal{Y}_{k+m}\right\}$, where $g^{m}(\cdot, \cdot)$ denotes the composition of $g m$-times. It is then easy to see that Theorem 3 remains valid.

Note that $\mathcal{P}_{k, k+m}$ is the projection on $\mathbb{R}^{n_{p}}$ of the higherdimensional set $F\left(\mathcal{P}, \mathcal{Y}_{k}, \mathcal{Y}_{k+m}\right) \in \mathbb{R}^{(m+1) n_{x}+2 n_{y}+n_{p}}$ defined by the system

$$
\begin{cases}y[k]=h(x[k], p) & \\ y[k+m]=h(x[k+m], p) & \\ x[i]=g(x[i-1], p) & i \in M \backslash\{k\} \\ x[i] \in \mathcal{X} & i \in M \\ y[k] \in \mathcal{Y}_{k} & \\ y[k+m] \in \mathcal{Y}_{k+m} & \\ p \in \mathcal{P}, & \end{cases}
$$

where $M=\{k, \ldots, k+m\}$. We denote as feasibility problem for $\mathcal{Y}_{k}, \mathcal{Y}_{k+m}$ the problem of checking whether $F\left(\mathcal{P}, \mathcal{Y}_{k}, \mathcal{Y}_{k+m}\right)=\emptyset$. An efficient approach to the solution of the feasibility problem (3) for polynomial systems is provided in the next section.

Multiple Measurements It is important to note that the equation system (3) could be easily extended to consider multiple measurements at once, simply including the constraints corresponding to the intermediates. As the number of measurements increases, this approach quickly becomes computationally prohibitive. Therefore, we now consider a less demanding, though more conservative, approach to model invalidation allowing to consider an arbitrary number of measurements. Key idea is to split the overall problem into a number of smaller problems and subsequently to infer the overall solution.

Consider the scheme depicted in Figure 1, where the measurements are indicated by error bars. Here, $\mathcal{P}_{i, j} \subseteq \mathcal{P}$ indicate the feasible parameter sets reproducing output trajectories being pairwise consistent with the measurements $\mathcal{Y}_{i}$ and $\mathcal{Y}_{j}$. Necessarily, if one of the parameter sets $\mathcal{P}_{i, j}$ is empty, then the model (2) is invalid. We additionally require a consistent parametrization of the model (2), defined as
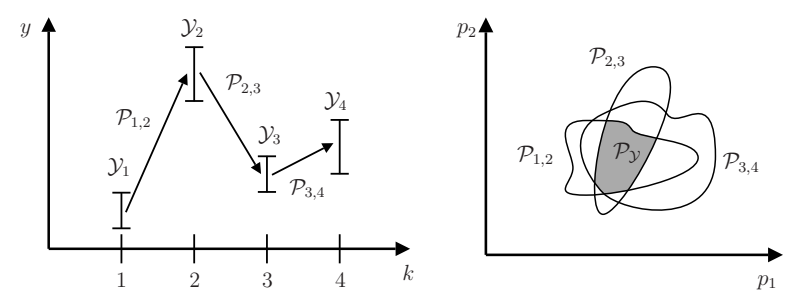

Fig. 1. Description of the invalidity approach. If the intersection of the feasible parameter sets is the empty set, then the model is invalidated by the measurements.

$$
P_{\mathcal{Y}}=\bigcap_{i=0}^{N-1} P_{i, i+1} .
$$

Note that $P_{\mathcal{Y}}$ provides an estimate of the model parameters. Thus, a strict criteria for model invalidity considering multiple measurements is given by:

Corollary 4. (Model Invalidity). If $P_{\mathcal{Y}}=\emptyset$ then the model (2) and the admissible parameter set $\mathcal{P}$ are invalidated by the measurements $\mathcal{Y}$.

In the remainder, we show how to solve the feasibility problem given by the equations (3) for the considered system class. In particular, we provide an algorithm to bound the feasible parameter set by partitioning the parameter space. Via infeasibility certificates, introduced in the next section, it is possible to classify subsets of the parameter space in order to obtain an outer-approximate of $P_{\mathcal{Y}}$.

\section{BOUNDING FEASIBLE PARAMETER SETS}

In this section we focus on how to estimate the feasible parameter sets for the considered problem class. In general, the feasibility problem $F\left(\mathcal{P}, \mathcal{Y}_{k}, \mathcal{Y}_{k+m}\right)(3)$ is a non-convex NP-hard optimization problem. Non-convexity results from the nonlinearities of the model equations.

Kuepfer et al. [2007] proposed a method that allows to obtain some results on the infeasibility of polynomial optimization problem. By taking advantage of the polynomiality, it is possible to relax $F\left(\mathcal{P}, \mathcal{Y}_{k}, \mathcal{Y}_{k+m}\right)$ to a convex semidefinite program (SDP). Semidefinite programs can be solved efficiently, e.g. via interior point methods.

To do so, the original feasibility problem is to be reformulated first as a quadratic feasibility problem (QFP). For simplicity of notation, let us consider two measurements taken at consecutive time steps. Hereafter, a vector $\xi$ is constructed containing the monomials of $x[k+1]-$ $g(x[k], p)$ and $y[k]-h(x[k], p)$, e.g.

$$
\xi^{T}=\left(1, p_{i}, x_{j}[k], p_{i} x_{j}[k], x_{j}[k+1], y_{l}[k]\right)
$$

for all $i \in N_{p}, j \in N_{x}, l \in N_{y}$, with $N_{p}=\left\{1, \ldots, n_{p}\right\}$, $N_{x}=\left\{1, \ldots, n_{x}\right\}$ and $N_{y}=\left\{1, \ldots, n_{y}\right\}$.

Let $S^{n}$ be the set of real symmetric $n \times n$ matrices, and let $\succeq$ denote the order operator with respect to the cone of positive semidefinite (PSD) matrices in $S^{n}$. The equality constraints $x_{i}[k+1]-g_{i}(x[k], p)=0$ and $y[k]-$ $h(x[k], p)=0$ can be written as 


$$
\begin{array}{r}
0=x_{i}[k+1]-g_{i}(x[k], p)=\xi^{T} Q_{i} \xi, i \in N_{x} \\
0=y_{i}[k]-h_{i}(x[k], p)=\xi^{T} R_{i} \xi, i \in N_{y}
\end{array}
$$

whereas $Q_{i}, R_{i}$ are constant symmetric matrices $\left(Q_{i}, R_{i} \in\right.$ $\left.S^{n_{\xi}}\right)$. Note also that some elements of the vector $\xi$ may be dependent from one another. Such dependencies can also be expressed quadratically, i.e.

$$
\xi^{T} D_{i} \xi=0, i \in N_{d}
$$

where $N_{d}=\left\{1, \ldots, n_{d}\right\}, n_{d}$ being the number of dependencies. The feasibility problem (3) can then be written as

$$
Q\left(\mathcal{P}, \mathcal{Y}_{k}, \mathcal{Y}_{k+1}\right):= \begin{cases}\text { find } \xi \in \mathbb{R}^{n_{\xi}} & \\ \text { s.t. } \xi^{T} Q_{i} \xi=0 & i \in N_{x} \\ \xi^{T} R_{i} \xi=0 & i \in N_{y} \\ \xi^{T} D_{i} \xi=0 & i \in N_{d} \\ B \xi \geq 0 & \\ \xi_{1}=1, & \end{cases}
$$

where $B \in \mathbb{R}^{n_{\xi} \times 2\left(n_{\xi}-1\right)}$ is constructed to cover the remainder of constraints, i.e. $\left(\mathcal{Y}_{k}\right.$ and $\left.\mathcal{Y}_{k+1}\right)$, the parameter region $\mathcal{P}$, and restrictions of the state space $x_{i}[k] \in \mathcal{X}$.

The obtained QFP can subsequently be relax to a SDP (Parrilo [2003]). The relaxation is found by setting $X=\xi$. $\xi^{T}$ and replacing the condition $\operatorname{rank}(X)=1$ and $\operatorname{tr}(X) \geq$ 1 with the weaker constraint $X \succeq 0$. This relaxation is based on an image convexification method (Ramana [1994]). The relaxed version of the QFP is thus obtained to as

$$
S D P\left(\mathcal{P}, \mathcal{Y}_{k}, \mathcal{Y}_{k+1}\right):= \begin{cases}\text { find } X \in S^{n_{\xi}} & \\ \text { s.t. } \operatorname{tr}\left(Q_{i} X\right)=0 & i \in N_{x} \\ \operatorname{tr}\left(R_{i} X\right)=0 & i \in N_{y} \\ \operatorname{tr}\left(D_{i} X\right)=0 & i \in N_{d} \\ B X e_{1} \geq 0 & \\ \operatorname{tr}\left(e_{1} e_{1}^{T}\right)=1 & \\ B X B^{T} \geq 0 & \\ X \succeq 0, & \end{cases}
$$

where $e_{1}=(1,0, \ldots, 0)^{T} \in \mathbb{R}^{n_{\xi}}$. Note that the relaxation is conservative. This guarantees that no solution is missed, but "false" solutions may have been introduced. This does not lead however to wrong invalidation results. In order to reduce the number of such wrong solutions, redundant constraints of the form $B X B^{T} \geq 0$ can be introduced (Kuepfer et al. [2007]).

It is a standard procedure in convex optimization to use the dual problem to certify infeasibility of the primal problem (Boyd and Vandenberghe [2004], Luenberger [2003]). Weak duality of SDP ensures that if the dual problem is unbounded, then the primal problem is infeasible, as shown by Waldherr et al. [2008]. The corresponding Lagrangian dual to $\operatorname{SDP}\left(\mathcal{P}, \mathcal{Y}_{k}, \mathcal{Y}_{k+1}\right)$ is given by

$$
L_{D}\left(\mathcal{P}, \mathcal{Y}_{k}, \mathcal{Y}_{k+1}\right)=\left\{\begin{array}{l}
\max \nu_{n_{\nu}} \\
\text { s.t. } B^{T} \lambda_{2} B+e_{1} \lambda_{1}^{T} B+B^{T} \lambda_{1} e_{1}^{T}+ \\
+\lambda_{3}+\sum_{i \in N_{x}} \nu_{i} Q_{i}+\sum_{i \in N_{y}} \nu_{n_{x}+i} R_{i}+ \\
+\sum_{i \in N_{d}} \nu_{n_{x}+n_{y}+i} D_{i}+\nu_{n_{\nu}} e_{1} e_{1}^{T}=0 \\
\lambda_{1} \geq 0, \lambda_{2} \geq 0, \lambda_{3} \succeq 0,
\end{array}\right.
$$

where $\lambda_{1} \in \mathbb{R}^{2\left(n_{\xi}-1\right)}, \lambda_{2} \in \mathbb{S}^{2\left(n_{\xi}-1\right)}, \lambda_{3} \in \mathbb{S}^{n_{\xi}}, \nu \in \mathbb{R}^{n_{\nu}}$ are the free variables, with $n_{\nu}=n_{x}+n_{y}+n_{d}+1$.

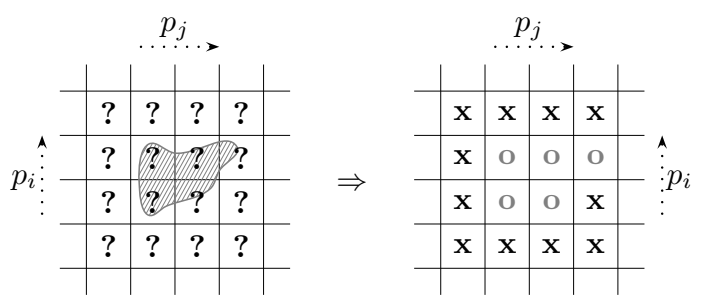

Fig. 2. Application of infeasibility certificates. The shaded area corresponds to the feasible parameter set. The symbol " $\mathrm{x}$ " denotes an infeasible partition, while "o" a feasible one.

\subsection{Estimation of Feasible Parameter Sets}

As infeasibility of (3) can be certified solving $L_{D}$, we can now outer-approximate the feasible parameter set by "cutting" off surrounding infeasible regions. The idea is depicted in Figure 2, where the shaded region encloses the feasible parameters for the problem.

This approach derives from the one proposed by Kuepfer et al. [2007], where parameter regions containing no steady states are similarly estimated via semidefinite programming. Our approach extends this result by taking not only the dynamical properties of the considered system into account, but also the model outputs.

In order to reduce the overall computational costs, the following bisection algorithm is implemented, as it allows to check groups of partitions simultaneously.

Algorithm 1. Outer-Approximate $\left(\mathcal{Q}, \mathcal{Y}_{k}, \mathcal{Y}_{k+1}\right)$

0 . If volume $(\mathcal{Q}) \leq \epsilon$, return $\mathcal{Q}$

1. Compute the Lagrangian dual $L_{D}\left(\mathcal{Q}, \mathcal{Y}_{k}, \mathcal{Y}_{k+1}\right)$

2. If $L_{D}$ is feasible, return $\emptyset$

3. If $L_{D}$ is not feasible, partition $\mathcal{Q}$ into $\mathcal{Q}_{1}$ and $\mathcal{Q}_{2}$

4. Set $\mathcal{Q}_{1}^{\prime}:=$ Outer-Approximate $\left(\mathcal{Q}_{1}, \mathcal{Y}_{k}, \mathcal{Y}_{k+1}\right)$

5. Set $\mathcal{Q}_{2}^{\prime}:=$ Outer-Approximate $\left(\mathcal{Q}_{2}, \mathcal{Y}_{k}, \mathcal{Y}_{k+1}\right)$

6. Return $\mathcal{Q}_{1}^{\prime} \cup \mathcal{Q}_{2}^{\prime}$

Note that $\mathcal{P}_{k, k+1}$ is contained in the set obtained calling Outer-Approximate $\left(\mathcal{P}, \mathcal{Y}_{k}, \mathcal{Y}_{k+1}\right)$, up to the given precision threshold $\epsilon$. This results in a robust and convergent way to explore the parameter space.

Remark 5. The computational time $T_{A}$ of Algorithm 1 is $O\left(N \cdot T_{D}\right)$, where $T_{D}$ is the time taken by a single Lagrangian dual computation and $N$ is the number of dual evaluations, which depends on the precision threshold considered in the algorithm. Reducing the threshold increases exponentially the time required, but it is important to note that the algorithm can be scheduled in parallel.

The proposed algorithm allows to estimate the feasible parameter sets. Next we show the application of our method to example systems.

\section{EXAMPLE}

As an example for an application of our approach, we consider two possible reaction mechanisms proposed by Henri [1902] between an enzyme (E) and a substrate (S) forming an enzyme-substrate complex (C): 


$$
E+S \underset{p_{-1}}{\stackrel{p_{1}}{\rightleftharpoons}} C \stackrel{p_{2}}{\longrightarrow} E+P,
$$

and

$$
C \underset{\tilde{p}-1}{\stackrel{\tilde{p}_{1}}{\rightleftharpoons}} E+S \stackrel{\tilde{p}_{2}}{\longrightarrow} E+P .
$$

These reaction schemes are known nowadays respectively as the Michaelis-Menten (MM) mechanism of enzyme activation (4) and as the Henri (H) mechanism (5). Both reaction schemes and their relevance are discussed in detail e.g. in Schnell et al. [2006], in which a main result states that both reaction mechanisms are analytically distinguishable if the transient initial dynamic of two independent states is considered. We therefore consider measurements in the transient phase.

The reaction mechanisms are modeled according to the law of mass action. Since both mechanisms obey the two conservation laws

$$
\begin{aligned}
& e_{0}=e(t)+c(t), \\
& s_{0}=s(t)+c(t)+p(t),
\end{aligned}
$$

both models can be expressed as second order systems. Let us then consider a simple first order explicit Euler discretization scheme (see e.g. Letellier et al. [2004]), given by

$$
\dot{x}[k] \approx \frac{x[k+1]-x[k]}{h},
$$

where $h>0$ denotes the size of the discretization step and $x[k]$ the value of $x(t)$ for $t=k h$. Fixing $e_{0}=1$, the corresponding difference equations for the MM mechanism are given by

$$
\begin{aligned}
& s[\mathrm{k}+1]=s[k]+p_{1} h\left((c[k]-1) s[k]+K_{S} c[k]\right) \\
& c[\mathrm{k}+1]=c[k]+p_{1} h\left((1-c[k]) s[k]-K_{M} c[k]\right),
\end{aligned}
$$

where $K_{S}=p_{-1} / p_{1}, K_{M}=\left(p_{-1}+p_{2}\right) / p_{1}$. For the Henri mechanism we obtain

$$
\begin{aligned}
& s[\mathrm{k}+1]=s[k]+h\left(\tilde{p}_{1}+\tilde{p}_{2}\right)\left((c[k]-1) s[k]-K_{H} c[k]\right) \\
& c[\mathrm{k}+1]=c[k]+\tilde{p}_{1} h\left((1-c[k]) s[k]-\tilde{K}_{S} c[k]\right),
\end{aligned}
$$

where $\tilde{K}_{S}=\tilde{p}_{-1} / \tilde{p}_{1}$ and $K_{M}=\tilde{p}_{-1} /\left(\tilde{p}_{1}+\tilde{p}_{2}\right)$.

\subsection{Model Discrimination}

In order to discriminate between both models, we consider measurements

$$
\mathcal{Y}^{H}=\{y: y[k]=(s[k], c[k]), 3 \leq k \leq 7\}
$$

taken from the Henri mechanism $(\mathrm{H})$ with $(s[0], c[0])=$ $(1,0), h=0.1 \mathrm{sec}$ and the parameter values $\tilde{p}_{1}=1$, $\tilde{p}_{-1}=1, \tilde{p}_{2}=1$. Next, we show that the MM is invalid with respect to these measurements.

For the invalidation setup, we consider as initial parameter space $\mathcal{P}^{M M}$ a range of one order of magnitude for each parameter:

$$
\frac{1}{3} \leq p_{1}, p_{-1}, p_{2} \leq 3 .
$$

In order to show model invalidity, the feasible parameter sets $\mathcal{P}_{3,4}, \mathcal{P}_{4,5}, \mathcal{P}_{5,6}$, and $\mathcal{P}_{6,7}$ for the corresponding time points are calculated via Algorithm 1. As partition of the parameter space $\mathcal{P}^{M M}$ we chose unitary hypercubes, whereas each cube is of size 0.05 . The feasible parameter sets are shown in Figure 3.

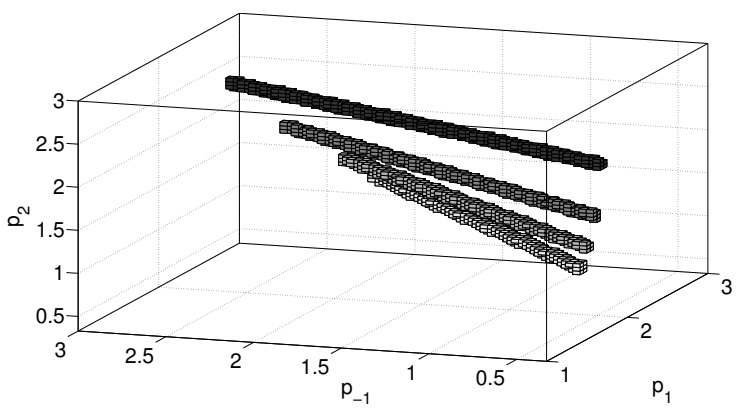

Fig. 3. Feasible parameter sets from $\mathcal{P}_{3,4}$ to $\mathcal{P}_{6,7}$ from to dark to light gray matching the measurements.

As can clearly be seen from the Figure 3, the intersection

$$
\mathcal{P}_{\mathcal{Y}}=\bigcap_{i=3}^{6} \mathcal{P}_{i, i+1}
$$

of the feasible parameter sets is empty. Thus, the Michaelis-Menten mechanism with the admitted $\mathcal{P}^{M M}$ is invalidated with respect to the measurements $\mathcal{Y}^{H}$.

\subsection{Parameter Estimation}

In order to estimate the model parameters, we consider now the measurements $y[k]=(s[k], c[k])^{T}, 1 \leq k \leq 6$ taken from the Michaelis-Menten mechanism (6) with $(s[0], c[0])=(1,0), h=0.1 \mathrm{sec}$ and the parameter values $p_{1}=1, p_{-1}=1, p_{2}=1$.

The feasible parameter sets $\mathcal{P}_{i, j}$ are estimated considering Algorithm 1 and depicted in Figure 4. In this case, the intersection $\mathcal{P} \mathcal{y}$ according to Corollary 4 is highlighted in black corresponding to the consistent feasible parameter set.

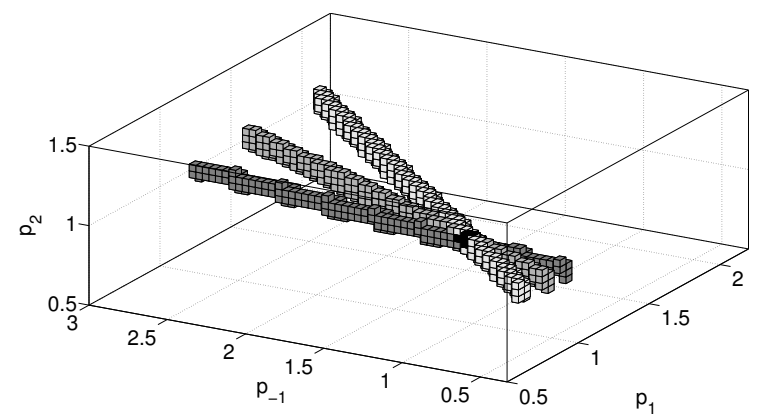

Fig. 4. Parameter sets. The intersection of the parameter sets is indicated black.

The complement $\mathcal{P} \backslash \mathcal{P}_{\mathcal{Y}}$ of $\mathcal{P}_{\mathcal{Y}}$ is therefore classified as infeasible.

As the number of measurements considered increases, the parameter estimates improve, see Figure 5. Notably, with this approach large regions of the parameter space can be discarded considering only a few measurements.

Remark 6. For the examples considered, the Lagrangian dual is computed within less than one second on a standard 

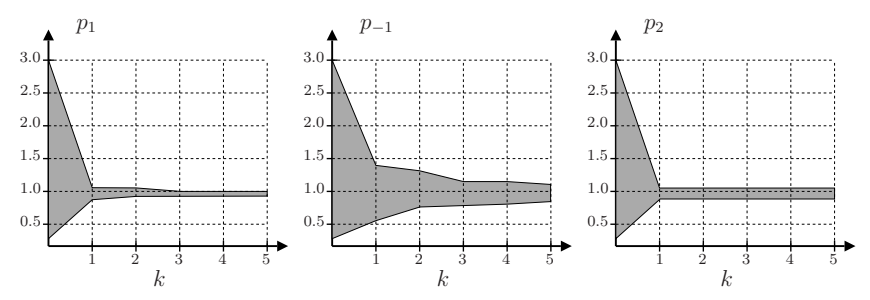

Fig. 5. Feasible parameter regions (shaded in the figure) with respect to the number of measurements taken into account.

desktop computer using MATLAB 2008 and the SEDUMI (Sturm [1999]) solver.

\section{DISCUSSION AND OUTLOOK}

We have studied the problems of model invalidation and parameter estimation of time-discrete polynomial systems with uncertain parameters. This is of particular importance for biochemical reaction networks, as it allows to discriminate between different model hypotheses. The proposed method provides conclusive results on the invalidation problem even if only imprecise or sparse measurements are available. It furthermore allows to discard large parameter regions, and therefore complements yet existing identification methods.

The proposed method is based on the outer-approximation of the parameter regions consistent with the experimental data. The parameter regions corresponds to the solution space of a nonlinear feasibility problem which can be relaxed to a semidefinite program. The Lagrangian dual of the SDP allows to efficiently bound the feasible parameter regions by a bisection algorithm. The derived method can be applied to a large class of nonlinear systems and can also be generalized and applied to state estimation as outlined in Borchers et al. [2009]. An extension to general nonlinear systems containing discrete variables can be done considering the same methods as in Hasenauer et al. [2009].

We have applied our methods to two possible enzymesubstrate reaction mechanisms. We were able to show invalidity of one of the mechanisms by considering data taken from the other one. We also demonstrated the applicability of our approach to obtain estimates on the model parameters.

In summary, our approach is a reliable and computationally manageable method for dynamical model invalidation and parameter estimation. Future work includes the extension of this approach to experimental design of biochemical reaction networks.

\section{ACKNOWLEDGMENTS}

The authors acknowledge funding by the research center "Dynamische Systeme in Biomedizin und Prozesstechnik" of the state Saxony-Anhalt, Germany and the Federal Ministry of Education and Research (BMBF-FRZ 0315280D).

\section{REFERENCES}

S. Borchers, P. Rumschinski, S. Bosio, R. Weismantel, and R. Findeisen. A set-based framework for coherent model invalidation and parameter estimation of discrete time nonlinear systems. 48th IEEE Conference on Decision and Control, CDC, 2009. Submitted.

S. Boyd and L. Vandenberghe. Convex Optimisation. Cambridge University Press, UK, 2004.

J. Chen and S. Wang. Structural indistinguishability between uncontrolled (autonomous) nonlinear analytic systems. IEEE Transactions on Automatic Control, 41(6):844-849, 1996.

A. Cornish-Bowden. Fundamentals of Enzyme Kinetics. Portland Press, 3rd edition, 2004.

I.R. Epstein and J.A. Pojman. An Introduction to Nonlinear Chemical Dynamics: Oscillations, Waves, Patterns, and Chaos. Oxford University Press, 1998.

N.D. Evans, M.J. Chappell, M.J. Chapman, and K.R. Godfrey. Structural indistinguishability between uncontrolled (autonomous) nonlinear analytic systems. Automatica, 40:1947-1953, 2004.

J. Hasenauer, P. Rumschinski, S. Waldherr, S. Borchers, F. Allgöwer, and R. Findeisen. Guaranteed steadystate bounds for uncertain chemical processes. In Proc. Int. Symp. Adv. Control of Chemical Processes, ADCHEM'09, 2009. To appear.

V. Henri. General theory of action of certain hydrolases. C. R. H. Acad. Sci. Paris, 135:916-919, 1902.

L. Kuepfer, U. Sauer, and P.A. Parrilo. Efficient classification of complete parameter regions based on semidefinite programming. BMC Bioinformatics, 8:12, 2007.

C. Letellier, S. Elaydi, L.A. Aguirre, and A. Alaoui. Difference equations versus differential equations, a possible equivalence for the Rössler system? Physica D, 195:29$49,2004$.

D.G. Luenberger. Linear and Nonlinear Programming. Springer, 2003.

P.A. Parrilo. Semidefinite programming relaxations for semialgebraic problems. Math. Program., Ser. B, 96: 293-320, 2003.

K. Poolla, P. Khargonekar, A. Tikku, J. Krause, and K. Nagpal. A time-domain approach to model validation. IEEE Transactions on Automatic Control, 39(5): 951-959, 1994.

S. Prajna. Barrier certificates for nonlinear model validation. Automatica, 42:117-126, 2006.

M.V. Ramana. An Algorithmic analysis of multiquadratic and semidefinite programming problems. $\mathrm{PhD}$ thesis, John Hopkins University, 1994.

C.P. Robert and G. Casella. Monte Carlo Statistical Methods. Springer, 2004.

S. Schnell, M.J. Chappell, N.D. Evans, and M.R. Roussel. The mechanism distinguishability problem in biochemical kinetics: The single-enzyme, single-substrate reaction as a case study. Comptes rendus-Biologies, 329: 51-61, 2006.

R.S. Smith and J.C. Doyle. Model validation: A connection between robust control and identification. IEEE Trans. Aut. Cont., 37(7):942-952, 1992.

J.F. Sturm. Using SeDuMi 1.02, a Matlab toolbox for optimization over symmetric cones. Optimization Methods and Software, 11:625-653, 1999.

S. Waldherr, R. Findeisen, and F. Allgöwer. Global sensitivity analysis of biochemical reaction networks via semidefinite programming. Proceedings of the 10th IFAC World Congress, 2008. 